\title{
Microvesicle-delivery miR-150 promotes tumorigenesis by up-regulating VEGF, and the neutralization of miR-150 attenuate tumor development
}

\author{
Yuchen Liu ${ }^{1}$, Luming Zhao ${ }^{1}$, Dameng Li ${ }^{1}$, Yuan Yin ${ }^{2}$, Chen-Yu Zhang ${ }^{1 凶}$, Jing Li ${ }^{1 \bowtie}$, Yujing Zhang ${ }^{1 凶}$ \\ ${ }^{1}$ Jiangsu Engineering Research Center for MicroRNA Biology and Biotechnology, State Key Laboratory of Pharmaceutical \\ Biotechnology, School of Life Sciences, Nanjing University, Nanjing 210093, China \\ 2 Oncology Institute, Fourth Affiliated Hospital of Suzhou University, Wuxi 214062, China \\ $\triangle$ Correspondence: yjzhang@nju.edu.cn (Y. Zhang), jingli220@nju.edu.cn (J. Li), cyzhang@nju.edu.cn (C.-Y. Zhang) \\ Received September 24, 2013 Accepted October 14, 2013
}

\begin{abstract}
Tumor-associated macrophages (TAMs) mostly exhibit M2-like (alternatively activated) properties and play positive roles in angiogenesis and tumorigenesis. Vascular endothelial growth factor (VEGF) is a key angiogenic factor. During tumor development, TAMs secrete VEGF and other factors to promote angiogenesis; thus, anti-treatment against TAMs and VEGF can repress cancer development, which has been demonstrated in clinical trials and on an experimental level. In the present work, we show that miR150 is an oncomir because of its promotional effect on VEGF. MiR-150 targets TAMs to up-regulate their secretion of VEGF in vitro. With the utilization of cell-derived vesicles, named microvesicles (MVs), we transferred antisense RNA targeted to miR-150 into mice and found that the neutralization of miR-150 down-regulates $m i R-150$ and VEGF levels in vivo and attenuates angiogenesis. Therefore, we proposed the therapeutic potential of neutralizing miR-150 to treat cancer and demonstrated a novel, natural, microvesicle-based method for the transfer of nucleic acids.
\end{abstract}

KEYWORDS microvesicle, miR-150, tumorigenesis, VEGF, neutralization, attenuation

\section{INTRODUCTION}

Tumors have a complex cellular ecology. Previous studies have shown that tumors consist not only of malignant cells but also of various stromal cell types (Lewis et al., 1995; Hanahan and Coussens, 2012). The stromal cells in cancer can be divided into three subtypes: angiogenic vascular cells (AVCS), infiltrating immune cells (IICs), and cancer-associated fibroblastic cells (CAFs) (Hanahan and Coussens, 2012). Tumor growth is clearly dependent on angiogenesis, as tumors need to establish a blood vessel network to access oxygen and nutrients (Millan Nunez-Cortes, 1991; Cristofanilli et al., 2002; Folkman, 2007). The contributions of stromal cells include resisting cell death, avoiding immune destruction, activating invasion, inducing angiogenesis, and sustaining proliferative signaling (Hanahan and Coussens, 2012).

The macrophages found in tumors are usually termed tumor-associated macrophages (TAMs) (Lewis and Pollard, 2006). The general understanding is that macrophages are subdivided into M1 (classically activated) and M2 (alternatively activated) phenotypes (Murdoch et al., 2008). M1 macrophages show a pro-inflammatory character, while M2 macrophages exhibit an immunosuppressive phenotype. TAMs often express many genes similar to those found in macrophages with the M2 phenotype and are thought to be M2-like macrophages (Murdoch et al., 2008). A number of studies have shown that TAMs influence tumor angiogenesis (Qian and Pollard, 2010; Fukuda et al., 2012). In human tumors, TAMs accumulate in poorly vascularized, necrotic areas and are also found surrounding blood vessels (Ferrara, 2010). A large number of publications have indicated that high numbers of TAMs are a poor prognostic indicator in cancer (Bingle et al., 2002). Lin and colleagues showed that decreasing macrophage infiltration in tumors resulted in the inhibition of tumor angiogenesis 
and delayed tumor development (Lin and Pollard, 2007). Further studies have found that TAMs express a broad range of pro-angiogenic factors that positively regulate angiogenesis, including vascular endothelial growth factor (VEGF) (Bolat et al., 2006), basic fibroblast growth factors (bFGF) (Bolat et al., 2006), tumor necrosis factor (TNF- $\alpha$ ), and transforming growth factor- $\beta$ (TGF- $\beta$ ) (Luo et al., 2006). These TAM-secreted factors explain the role of TAMs in tumor development. Because of their pivotal role in tumor growth, TAMs and their factors have been considered to be anticancer therapeutic targets. The blockade of colony stimulating factor-1 (CSF-1) signaling resulted in macrophage depletion in mammary tumors, reduced vascular density and improved responses to chemotherapy (Lin et al., 2001). Histidine-rich glycoprotein (HRG) can induce the reprogramming of TAMs, which has been shown to normalize vascular formations, resulting in a decrease in torturous vasculature, better pericyte coverage, less erratic blood flow, and improved responses to chemotherapy (Rolny et al., 2011).

VEGF is an angiogenic factor that can bind to receptors to induce endothelial cell migration and form new blood vessels (Ferrara et al., 2003). TAM-derived VEGF acts significantly on angiogenesis and tumor development, whereas down-regulating VEGF levels induces antitumorigenesis (Bolat et al., 2006). The deletion of the VEGF gene in macrophages decreases tumor angiogenesis (Stockmann et al., 2008). Anti-bodies against VEGF exert a large inhibitory effect on tumor growth (Kim et al., 1993). Cancer patients can currently receive antiVEGF treatment, which results in a greater anti-tumor effect.

miRNAs are a class of small, non-coding RNAs that regulate gene expression at the post-transcriptional level (Bartel, 2004). Previous studies have demonstrated that miRNAs are relevant to cancers (Esquela-Kerscher and Slack, 2006), and anti-miRNA treatments have demonstrated anti-tumor effects on an experimental level (Kota et al., 2009). Previous study has found that miRNAs can be packaged into microvesicles (MVs), which are membranous vesicles that are secreted from most cell types, and transferred to neighboring or distant cells, where these miRNAs can regulate the functions of the recipient cells (Zhang et al., 2010). MVs have been utilized to transfer therapeutic RNAi to treat diseases (Alvarez-Erviti et al., 2011). In our present study, we demonstrated that miR-150 serves as an oncomir by regulating the VEGF secretion of TAMs. Then, MVs were utilized as the means for delivering antisense RNA targeted to miR-150 into mice to treat tumors and decrease tumor development by influencing the level of VEGFs secreted by TAMs. Our present study proposed a new anti-tumor molecule and a new method for the transfer of antineoplastic drugs.

\section{RESULTS}

Microvesicle-delivered miR-150 promotes tumor development in vivo

MiR-150 is an immune-related miRNA that is up-regulated in the plasma with the occurrence of several types of cancers (Fig. 1A). Thus, we hypothesized that miR-150 plays a role in

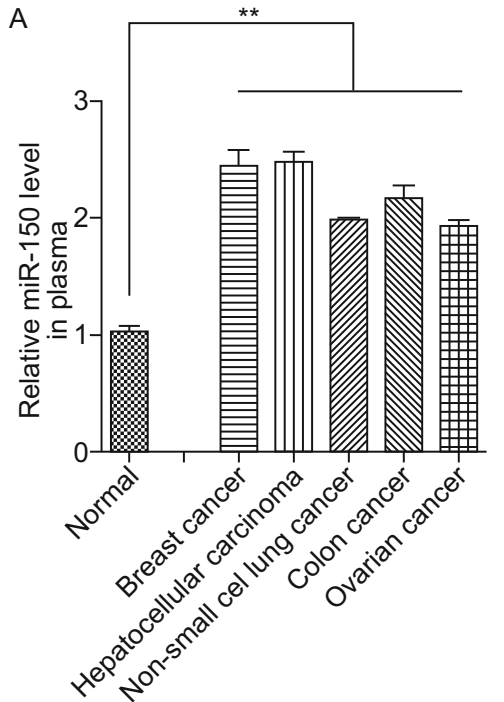

B

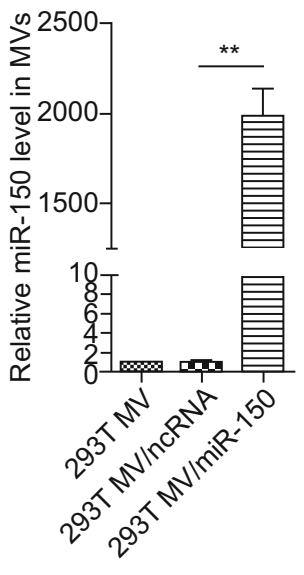

Figure 1. Microvesicle-delivered miR-150 promotes tumor development in vivo. (A) qRT-PCR analysis of the miR-150 levels in plasma from normal controls and patients with breast cancer, hepatocellular carcinoma, non-small cell lung cancer, colon cancer or ovarian cancer $(n=4)$. (B) qRT-PCR analysis of the miR-150 levels in MVs derived from 293T cells, 293T cells transfected with miRNA mimic negative control and 293T cells transfected with miR-150 mimic $(n=4)$. *,$P<0.05 ;{ }^{* *}, P<0.01$.

tumorigenesis. To verify our hypothesis, we conducted the following experiments. We delivered miR-150 into mice using microvesicles to assess the effect of miR-150 on tumorigenesis. The human embryo kidney cell line 293T and its MVs contain a very low level of miR-150 (Zhang et al., 2010); thus, 293T MVs were chosen to serve as the carrier for miR-150. MiR-150 was over-expressed in the 293T cell line by transfecting mimicmiR-150 RNA; non-sense RNAs were also transfected into 293T cells to serve as the negative control. After transfection, MVs from 293T cells transfected with mimic-miR-150 RNA contained much higher levels of miR-150 compared with MVs from 293T cells transfected with ncRNA (Fig. 1B). Mice were subcutaneously implanted with sarcoma cells (S-180) to create a tumor model, and 293T MVs with or without miR-150 were intravenously injected into the tumor mice models as described in Fig. 2A. The results showed that the injection of 293T MVs containing miR-150 increased the plasma miR-150 levels (Fig. 2B). Tumor weight also dramatically increased after treatment with miR-150 (293T MV/miR-150) when compared with the saline group and the 293T/ncRNA group (Fig. 2C and 2D), suggesting that 293T MV-delivered exogenous miR-150 promotes tumor development in vivo.

VEGF is a very crucial factor in the process of tumor development. Tumors secrete VEGF to induce endothelial cell migration and the formation of new blood vessels. Serum VEGF levels were also assessed after the injection of miR150. The ELISA results showed that VEGF levels increased in 
A

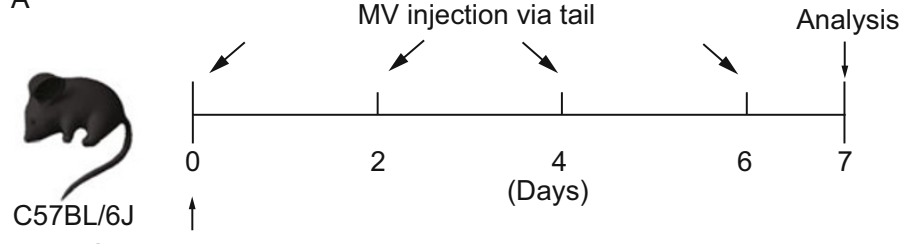

S-180 implanted

C

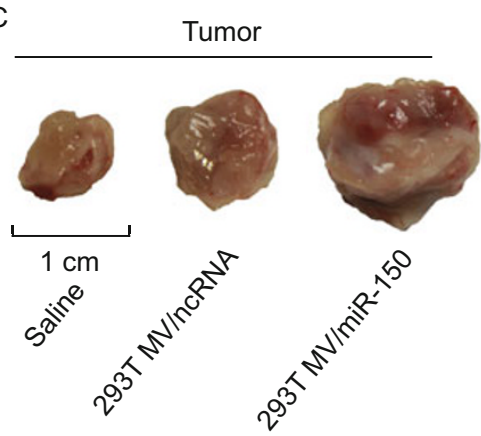

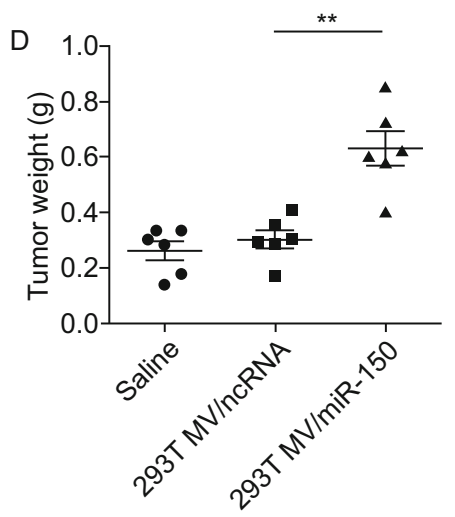

B

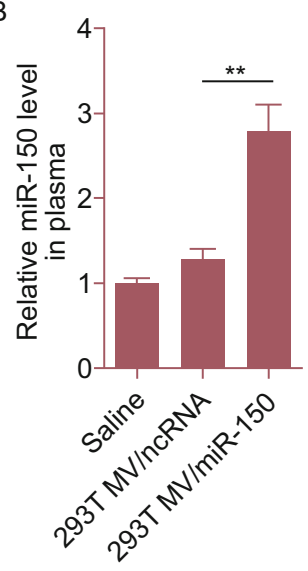

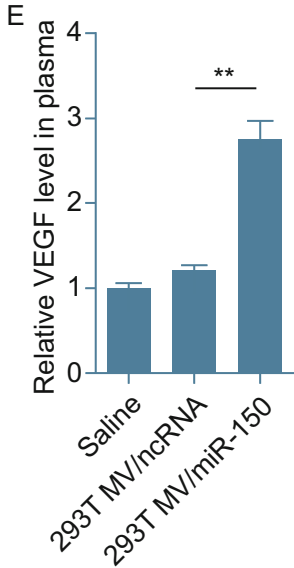

Figure 2. Microvesicle-delivered miR-150 promotes tumor development in vivo. (A) Flow chart depicting the experimental design. The C57BL/6J mice were first implanted with S-180 sarcoma cells and injected via the tail vein with saline (control), normal or modified 293T MVs (named 293T MV/miR-150) once every two days. The modified 293T MVs were harvested by transfecting the cells with the miR-150 mimic. Seven days later, the tumor tissues were analyzed and tumor sizes were measured. (B) qRT-PCR analysis of the plasma miR-150 levels in tumor-implanted mice that were injected with saline (control), normal 293T MVs or 293T MV/miR-150 ( $n=8)$. (C) Morphology of the tumor tissues excised from tumor-implanted mice that were injected with saline (control), normal 293T MVs or 293T MVI miR-150. (D) Weight of the tumor tissues excised from tumor-implanted mice that were injected with saline (control), normal 293T MVs or 293T MV/miR-150. (E) ELISA analysis of the plasma VEGF levels in tumor-implanted mice that were injected with saline (control), normal 293T MVs or 293T MV/miR-150 $(n=8) .{ }^{*}, P<0.05 ;{ }^{* *}, P<0.01$

the plasma following the injection of miR-150 (Fig. 2E). Taken together, these results demonstrate that miR-150 promoted tumor development and suggest that the promotion of tumor development may be mediated by VEGF.

\section{MiR-150 targets TAMs and promotes VEGF secretion by TAMs}

To further assess whether miR-150 has a positive effect on VEGF secretion, we performed an in vitro assay. Considering that most TAMs are thought to be M2-like and that TAMs are the major origin of VEGF during tumor development, we hypothesized that miR-150 may target TAMs and consequently alter the levels of VEGF secreted. To validate our hypothesis, the effect of miR-150 on TAMs was assessed. TAMs were isolated from mice sarcoma tissue. To verify whether TAMs from the sarcoma were M2-like macrophages, bone marrow cells were isolated from the femur and were polarized into M1 and M2 macrophages, which were used as the reference cells for TAMs. Markers that can efficiently distinguish between M1 and M2 macrophages were tested. As we expected, the expression of these markers in TAMs was similar to M2 macrophages and differed from those observed in M1 macrophages, suggesting that the sarcoma-derived TAMs were M2-like macrophages (Fig. 3A). MiR-150 is abundantly expressed in MVs derived from THP-1 human monocyte cells following AGE treatment (Fig. S1A). Thus, to examine the effect of miR-150 on TAMs, AGE-treated THP-1 MVs that were rich in miR-150 were incubated with TAMs for $8 \mathrm{~h}$, after which the medium was collected for use in an ELISA assay. The ELISA results showed that THP-1 MVs (AGE) dramatically enhanced VEGF secretion from TAMs (Fig. 3B). Although miR-150 is abundantly expressed in THP-1 MVs, other contents in the MVs may be responsible for the promotion of VEGF secretion. To solve this problem and confirm the role of miR-150, the following experiment was conducted. We knocked down miR-150 in THP-1 MVs by transfecting THP-1 cells with antisense oligonucleotides against miR-150 (anti-miR-150); ncRNAs were also transfected into THP-1 cells as a negative control. The transfected THP-1 cells were treated with AGE before medium collection. The results showed that miR-150 expression was reduced in the modified THP-1 MVs (Fig. S1B). TAMs were incubated with these miR-150-deficient MVs. After an $8 \mathrm{~h}$ incubation, the results revealed that the miR-150-deficient MVs (THP-1 MV/ miR-150 def) lost the ability to enhance VEGF secretion when compared to THP-1 MVs loaded with ncRNAs (THP-1 MV/ anti-ncRNA), suggesting that the promotion of VEGF secretion was mediated by miR-150 but not by other contents in the MVs (Fig. 3C). Taken together, these results indicated that miR-150 

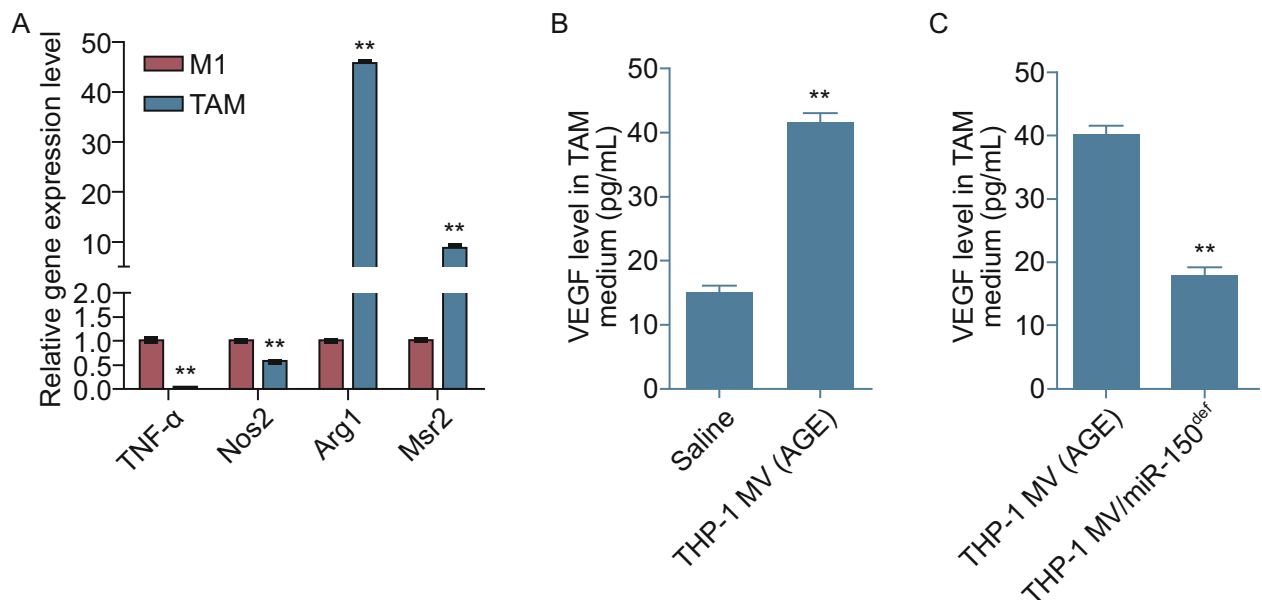

Figure 3. MiR-150 targets TAMs and promotes VEGF secretion by TAMs. (A) qRT-PCR analysis of the relative gene expression levels of TNF- $\alpha$, NOS1, Arg2, and Msr2 of M1 macrophages or TAMs. (B) ELISA analysis of the VEGF levels in the medium of TAMs treated with saline (control) or THP-1 MV (AGE treated). (C) ELISA analysis of the VEGF levels in the medium of TAMs treated with normal THP-1 MV or miR-150 deficient MV (THP-1 MV/miR-150 def). * $P<0.05 ;{ }^{* *}, P<0.01$.

increased the VEGF secretion of TAMs, resulting in a positive effect on tumor development.

\section{Microvesicle-delivered miR-150 inhibitor abolishes tumorigenesis and is proposed to be a potential therapeutic molecule against tumors}

Because high levels of miR-150 promote tumor development, neutralizing miR-150 could potentially attenuate tumorigenesis. Taking advantage of the low level of miR-150 in 293T cells and 293T MVs, 293T MVs could be used as a carrier to transfer antisense RNA against miR-150 (anti-miR-150) into tumor models. We transfected 293T cells with antisense miR150 RNA to load the MVs with the miR-150 inhibitor (293T MV/anti-miR-150). Mice were subcutaneously implanted with S-180 sarcoma cells to create a tumor model, and we treated this model with 293T MVs loaded with the miR-150 inhibitor or ncRNA, as depicted in Fig. 4A. The plasma miR-150 levels in mice treated with anti-miR-150 (293T MV/anti-miR-150) decreased significantly compared with those from the ncRNA group and the control group (Fig. 4B), indicating that the miR150 inhibitor effectively decreased the plasma miR-150 levels of the tumor models. The VEGF levels in the plasma and tumor tissue also decreased when mice were treated with miR150 antisense RNA (Fig. 4C). Subsequently, the tumor weights from the 293T MV/anti-miR-150 group dramatically decreased (Fig. 4D and 4E), suggesting that tumor development was strongly restrained. Consistent with the previous result, the protein levels of VEGF in xenograft decreased dramatically with the treatment of miR-150 antisense RNA (Fig. 4F). The results of the HE staining of paraffin sections showed that tumors treated with the miR-150 inhibitor presented large areas of necrosis because of fewer palingenetic blood vessels (Fig. 4G), further suggesting that angiogenesis and tumor development had been inhibited by the miR-150 inhibitor injection. The results of the immunohistochemical staining of paraffin sections revealed that the expression levels of the endothelial cell marker CD31 were dramatically reduced after miR-150 inhibitor treatment (Fig. $4 \mathrm{H}$ ), suggesting that the migration of endothelial cells into the tumor was inhibited and that angiogenesis was inhibited in the tumor. Taken together, these results demonstrated that the down-regulation of miR-150 in a tumor model efficiently inhibited VEGF secretion and consequently restrained tumor development, suggesting that miR-150 could be a potential therapeutic target in cancers and that MV-delivered anti-miR-150 could be a novel strategy to treat cancers.

\section{Microvesicle-delivered miR-150 inhibitor prevents tumorigenesis}

We also found that the inhibitor of miR-150 played a long-term function in regulating tumorigenesis in vivo. The experiment was conducted as depicted in Fig. 5A. The MVs containing the inhibitor of miR-150 were injected into mice via the tail vein prior to the mice being implanted with sarcoma cells. After 7 days, the plasma miR-150 and VEGF levels decreased dramatically in mice receiving miR-150 inhibitor treatment (293T MV/antimiR-150) compared with mice treated with saline or ncRNA (293T MV/anti-ncRNA) (Fig. 5B and 5C). The tumor weights from the anti-miR-150 injection group (293T MV/anti-miR-150) were lower than those from mice that were injected with 293T MV/ncRNA or saline (Fig. 5D and 5E). The result of the HE staining of paraffin sections showed a decrease in angiogenesis in tumors from mice treated with the miR-150 inhibitor (293T MV/anti-miR-150) compared with those from the other two groups (Fig. 5F). The immunohistochemical staining of paraffin sections showed that CD31 expression was reduced in tumors from mice treated with the inhibitor of miR-150 (293T MV/anti- 
A

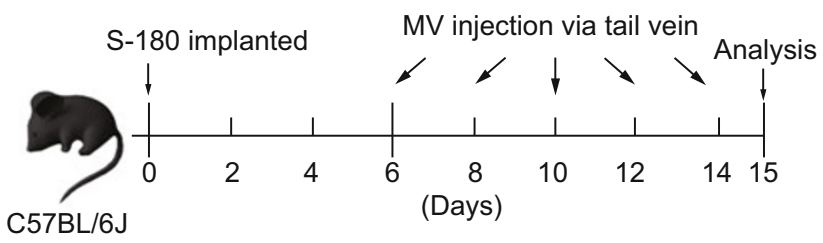

$57 \mathrm{BL} / 6$

D

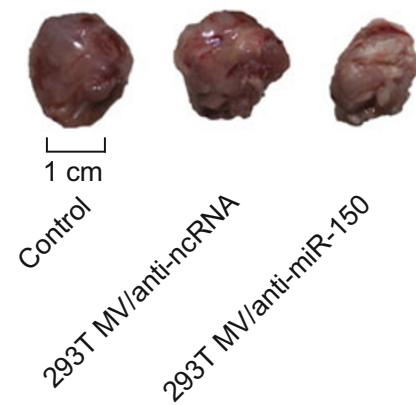

E

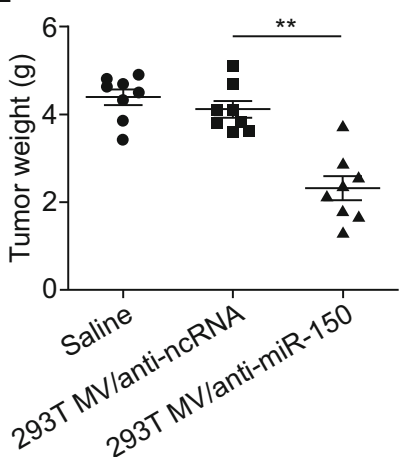

$$
\text { B }
$$

C

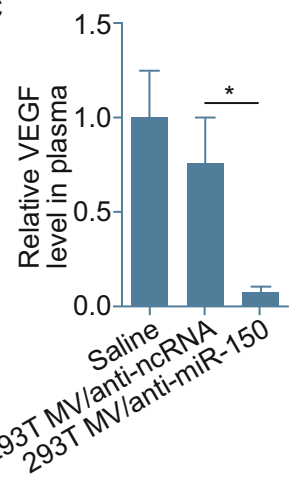

G

Control

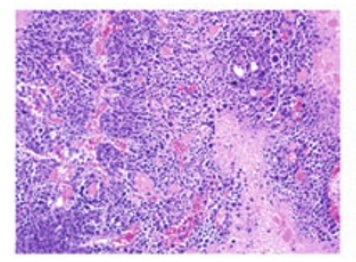

293T MV/anti-ncRNA

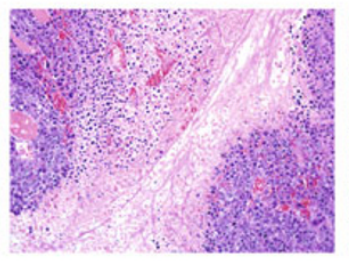

293T MV/anti-miR-150

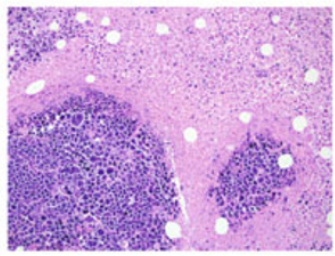$$
\text { ( }
$$
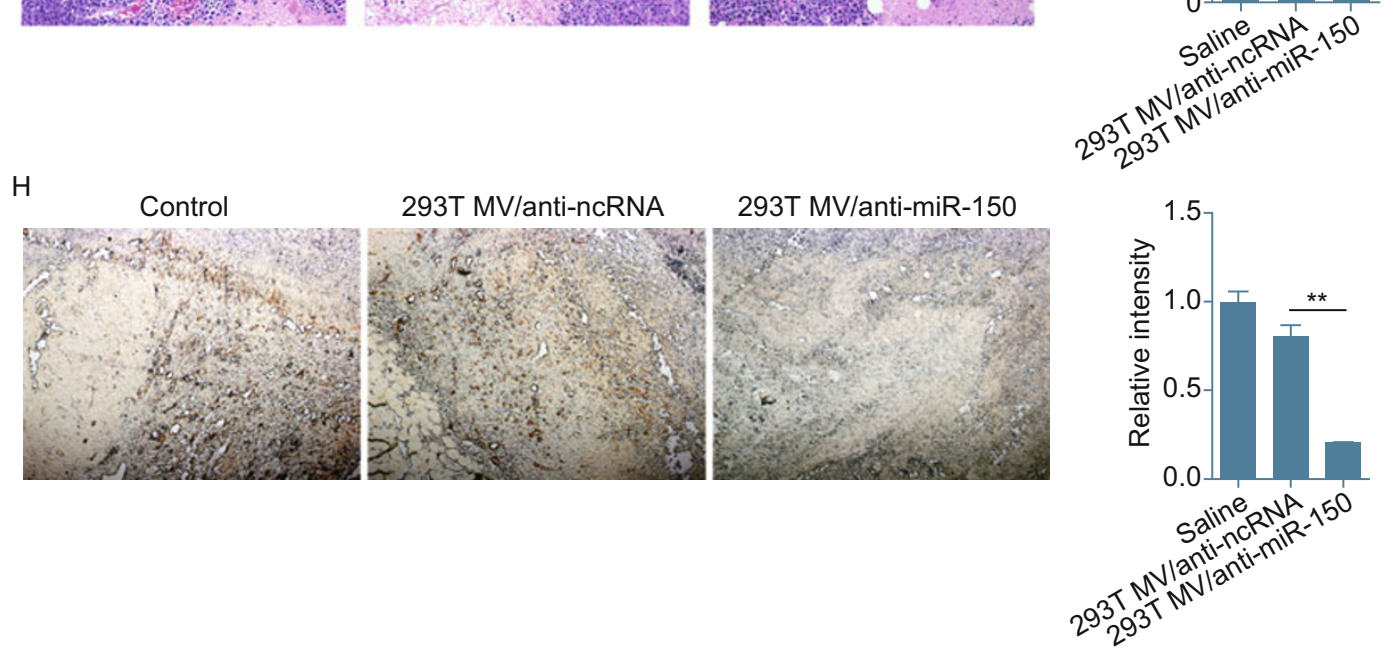

Figure 4. Microvesicle-delivered miR-150 inhibitor abolishes tumorigenesis and is proposed to be a potential therapeutic molecule for tumor treatment. (A) Flow chart depicting the experimental design. The C57BL/6J mice were first implanted with S-180 sarcoma cells. After 6 days, the mice were injected via the tail vein with saline (control) or with "modified" 293T MVs (named 293T MV/ncRNA or 293T MV/anti-miR-150) once every two days. The "modified" 293T MVs were harvested by transfecting the cells with negative control RNA or anti-miR-150. Nine days later, the tumor tissues were analyzed and tumor sizes were measured. (B) qRT-PCR analysis of the plasma miR-150 levels in tumor-implanted mice that were injected with saline (control), 293T MV/ncRNA or 293T MV/anti-miR-150 ( $n=8$ ). (C) ELISA analysis of the plasma VEGF levels in tumor-implanted mice that were injected with saline (control), 293T MV/ncRNA or 293T MV/anti-miR-150 ( $n=8)$. (D) Morphology of the tumor tissues excised from tumor-implanted mice that were injected with saline (control), 293T MV/ncRNA or 293T MV/anti-miR-150. (E) Weight of the tumor tissues excised from tumor-implanted mice that were injected with saline (control), 293T MV/ncRNA or 293T MV/anti-miR-150. (F) Representative image of Western blot analysis of xenograft VEGF from mice that were untreated or treated with 293T MV/ncRNA or 293T MV/anti-miR-150 $(n=8)$. (G) Left panel: H\&E stained sections from the tumor tissues shown in panel C, with arrows pointing to the luminal structures containing red blood cells. Right panel: Quantitative analysis of the numbers of newly formed vessels in tumor sections $(n=8)$. $(\mathrm{H})$ Left panel: Immunohistochemistry of the paraffin-embedded tumor tissues shown in Fig. 4C using a CD31 antibody. Right panel: Quantitative analysis of the intensity of CD31 in tumor sections $(n=8)$. ${ }^{*}, P<0.05 ;{ }^{* *}, P<0.01$. 
A

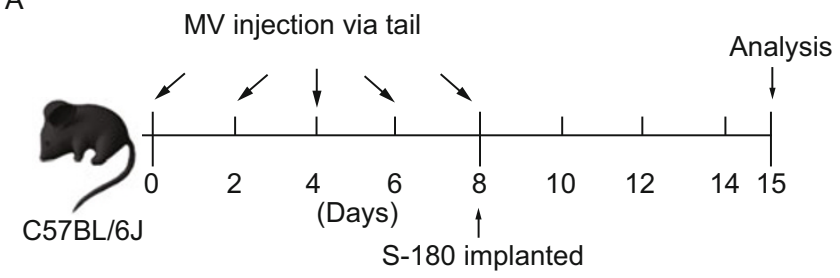

D

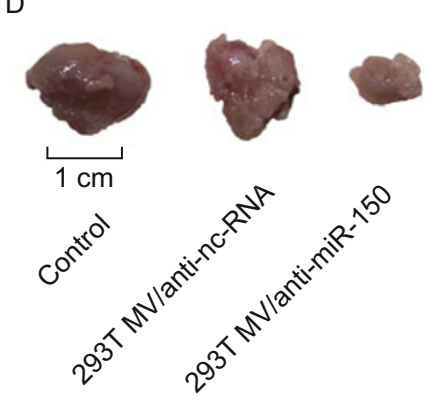

E

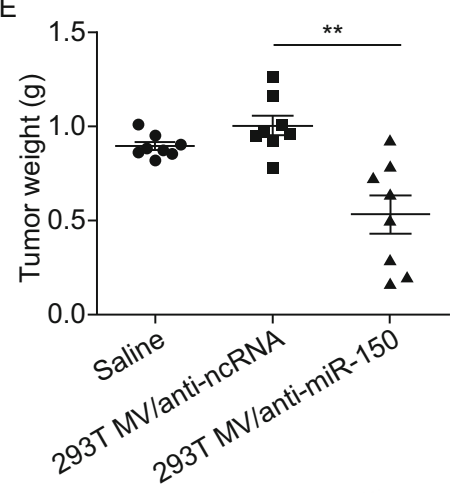

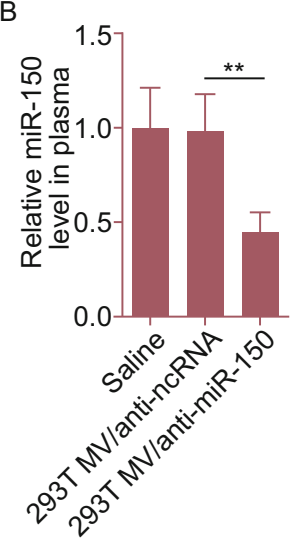

C

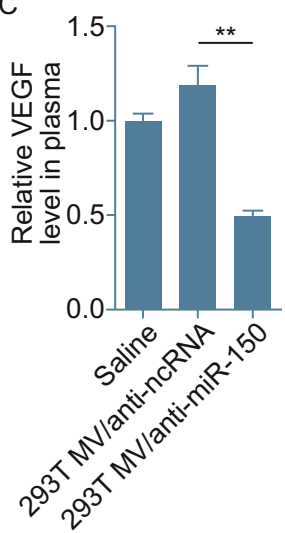

$\mathrm{F}$

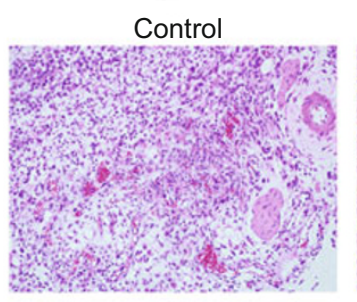

293T MV/anti-nc-RNA
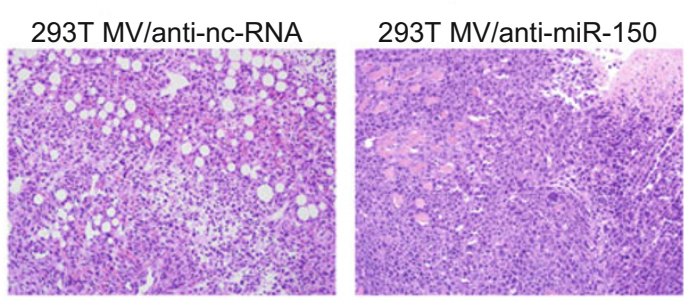

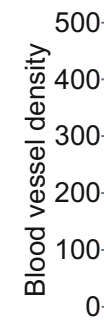

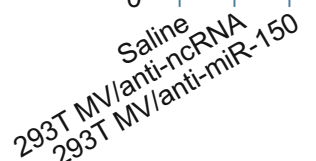

G

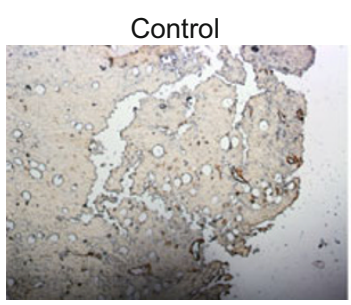

293T MV/anti-nc-RNA

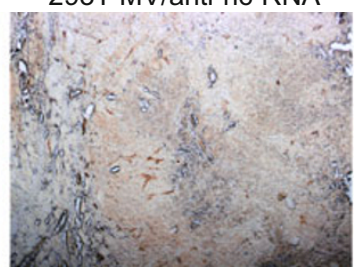

293T MV/anti-miR-150

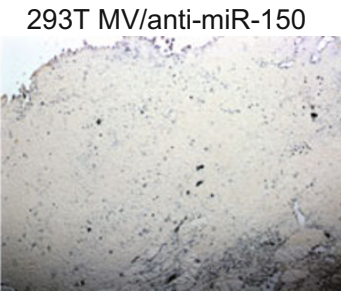

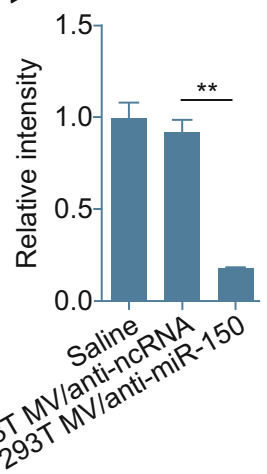

Figure 5. Microvesicle-delivered miR-150 inhibitor prevents tumorigenesis. (A) Flow chart depicting the experimental design. The C57BL/6J mice were first injected via the tail vein with saline (control) or "modified" 293T MVs (named 293T MV/ncRNA or 293T MV/ anti-miR-150) once every two days. The "modified" 293T MVs were harvested by transfecting the cells with negative control RNA or antimiR-150. After 8 days, the mice were implanted with S-180 sarcoma cells. Seven days later, the tumor tissues were analyzed and tumor sizes were measured. (B) qRT-PCR analysis of the plasma miR-150 levels in tumor-implanted mice that were injected with saline (control), 293T MV/ncRNA or 293T MV/anti-miR-150 $(n=8)$. (C) ELISA analysis of the plasma VEGF levels in tumor-implanted mice that were injected with saline (control), 293T MV/ncRNA or 293T MV/anti-miR-150 $(n=8)$. (D) Morphology of the tumor tissues excised from tumorimplanted mice that were injected with saline (control), 293T MV/ncRNA or 293T MV/anti-miR-150. (E) Weight of the tumor tissues excised from tumor-implanted mice that were injected with saline (control), 293T MV/ncRNA or 293T MV/anti-miR-150. (F) Left panel: H\&E stained sections from the tumor tissues shown in panel $\mathrm{C}$, with arrows pointing to the luminal structures containing red blood cells. Right panel: Quantitative analysis of the numbers of newly formed vessels in tumor sections $(n=8)$. (G) Left panel: Immunohistochemistry of the paraffin-embedded tumor tissues shown in Fig. 5C using a CD31 antibody. Right panel: Quantitative analysis of the intensity of CD31 in tumor sections $(n=8)$. ${ }^{*}, P<0.05 ;{ }^{* *}, P<0.01$. 
miR-150), suggesting that MV-delivered anti-miR-150 inhibited endothelial cell migration and angiogenesis in the tumors (Fig. 5G). Taken together, these results demonstrated that the preemptive neutralization of miR-150 may alter the microenvironment of tumors and prevent subsequent tumorigenesis. The inhibitor of miR-150, protected by MVs, may survive and contribute to the regulation of tumor development over a longterm period.

\section{DISCUSSION}

During tumor development, tumor cells secrete chemoattractants to ensure the ongoing recruitment of monocytes into tumors, including some types of growth factors, such as VEGF, placenta growth factor (PIGF), and CSF-1 (Coffelt et al., 2009). TAMs exhibit several characteristics of M2-polarized macrophages and promote tumor development by promoting angiogenesis and lymphangiogenesis, sustaining proliferative signaling and resisting cell death (Fukuda et al., 2012). Our present study focused on the role of TAMs in the regulation of angiogenesis, largely via their production of VEGF. MiRNAs are a class of classic molecule that regulates tumor development. The term oncomir is specifically used to reference miRNAs that have critical functions in tumor development (Goga and Benz, 2007). MiR-150 is an immune-related miRNA, and, for the first time, the present study has found that miR-150 promotes tumorigenesis (Fig. 2C). MiR-150 also promotes increased levels of VEGF in plasma (Fig. 2E). Considering the large role VEGF plays in the induction of angiogenesis and tumor development, the significant promotion of tumor growth by miR-150 may be mediated by increased VEGF levels. TAMs are a major source of VEGF, which binds to receptors on endothelial cells to promote migration and budding (Bolat et al., 2006). Thus, we proposed the hypothesis that TAMs may be the target cell of miR-150 during tumor development. In the present study, our results showed that exogenous miR-150 can increase the VEGF secretion by TAMs in vitro, revealing that miR-150 acts as a promotion factor in tumor development by regulating the VEGF secretion of TAMs (Fig. 3B and 3C). VEGF is not the direct target of miR-150, and miR-150 may target other upstream proteins involved in VEGF regulation. We predicted the target genes of miR-150 using Targetscan (http://www.targetscan.org/) and found that the inhibitor of growth family member 4 (ING4) is a very perfect target. It is well known that ING4 is a tumor suppressor for it intervenes the activity of hypoxia inducible factor (HIF) which has an important positive role in angiogenesis and tumorigenesis. HIF promote tumor angiogenesis by inducing VEGF and other factors (Ozer et al., 2005; Colla et al., 2007). Thus, the up-regulation of VEGF by miR-150 may be mediated by ING4 (Brahimi-Horn and Pouyssegur, 2006). Another predicted target of miR-150 by miRnada (http:// www.microma.org/) is OS9, which is an essential component of a multiprotein complex that regulates HIF1A levels in an oxygen-dependent manner (Baek et al., 2005). It is reported that knockdown of OS9 by RNA interference increased HIF1A protein levels, HIF1A transcriptional activity, and VEGF mRNA levels under nonhypoxic conditions (Baek et al., 2005). We also found that THP-1-derived MVs contained VEGF, which raised the issue of whether the VEGF in the MVs or miR-150 was responsible for the increased levels of VEGF. Through calculation, we found that the proportion of VEGF contained in the MVs was only $1.5 \%$ of the increased VEGF in medium, and the other $98.5 \%$ of increased VEGF was contributed by the effects of exogenous miR-150 (Fig. S1C).

Great progress has been made in developing therapeutic strategies that target TAMs and VEGF to treat cancer (Kim et al., 1993). Several studies have revealed that TAMs are a very critical cell group in tumor tissue and that TAMs contribute greatly to tumorigenesis and the protection of tumors from therapy (McDonnell et al., 2003). VEGF inhibition represses tumor angiogenesis, and this result has led to the clinical utilization of VEGF inhibitors to treat cancer (Warren et al., 1995; Presta et al., 1997). In our present study, we used a microvesicledelivered antagonucleotide against miR-150 to treat tumors. After the injection, VEGF levels decreased because of the down-regulation of miR-150 (Fig. 4C), and tumor growth was abolished by anti-miR-150 treatment (Fig. 4D). Through the angiogenic morphogenesis results, we found that angiogenesis was attenuated after antisense injection (Fig. 4F and 4G). Thus, we concluded that the miR-150 antisense caused the anesis of tumor development by down-regulating VEGF. This method provides a new, potential target to treat cancer, and the function of miRNAs in cancer development should not be ignored.

Microvesicles, a class of membrane vesicles with a diameter of 30-1000 nm, contain shedding vesicle and exosome (Chen et al., 2012). They are secreted by most cell types in vitro (Valadi et al., 2007). MVs have been utilized for the delivery of therapeutic RNAi and are considered to be a more effective, advantageous method than other options. Compared with the delivery strategies of viruses, lipid nanoparticles and polymeric nanoparticles, MVs present some major advantages. MVs are a natural carrier and are not subject to attack by antibodies, complements or opsonins in circulation. Other methods are prone to be cleared or trigger unwanted immune responses (van den Boorn et al., 2011). In our present study, MVs delivered the antagonucleotide for miR-150 into tumors efficiently and repressed tumor development, further suggesting that $\mathrm{MVs}$ are the perfect carrier for transferring therapeutic small RNA. Furthermore, we found that the function of the injected antisense continued for quite a long time. Seven days after the injection, miR-150 was maintained at a low level in the plasma, and VEGF levels and tumor development were also repressed (Fig. 5B-D), demonstrating that the antisense was still functional in vivo. In this process, MVs may act as a protector for the inner antisense and allow the antisense to exist and act for a long time.

An increasing body of research has revealed the critical role of miRNAs in the process of tumor development and demonstrated that miRNAs can serve as therapeutic targets. Our pre- 
sent study found that anti-miRNA treatment efficiently triggered the attenuation of tumor growth and suggests that MVs may be a potential carrier for the delivery of small nucleic acids. The utilization of MV-delivered small RNA may be a prospective therapeutic strategy.

\section{MATERIALS AND METHODS}

Cell lines

The human embryonic kidney 293T cell line and the human acute monocytic leukemia cell line THP-1 were purchased from the Shanghai Institute of Cell Biology, Chinese Academy of Sciences (Shanghai, China). The 293T cells were cultured in high-glucose DMEM medium, supplemented with $10 \%$ fetal bovine serum (FBS) (Gibco, CA, USA). The THP-1 cells were cultured in standard RPMI 1640 medium, supplemented with $10 \%$ FBS (Gibco).

Animals, tumor model generation, and primary cell isolation

C57BL/6J (male, 8-week-old) mice were purchased from the Model Animal Research Center (MARC) of Nanjing University (Nanjing, China) and were maintained in pathogen-free conditions. The animals received humane care according to the guidelines prepared by the $\mathrm{Na}-$ tional Institutes of Health's Guide for the Care and Use of Laboratory Animals and approved by the Institutional Review Board of Nanjing University, Nanjing, China.

To generate the xenograft tumor models, S-180 mouse sarcoma cells $\left(10^{6}\right.$ cells per mouse) were subcutaneously injected into the left armpits of the animals. The tumor sizes were measured using a caliper and the tumor weights were determined after the tumors were separated from the animals.

Bone marrows were isolated from 8-week-old mice by flushing their femurs. The cells were suspended and maintained in RPMl 1640 media, supplemented with $10 \%$ FBS (Gibco) and $800 \mathrm{pg} / \mathrm{mL}$ mice M-CSF. Bone marrow cells were differentiated into non-polarized macrophages (M0) after 7 days. M0 cells were treated with $1 \mu \mathrm{g} / \mathrm{mL}$ LPS and $20 \mathrm{ng} / \mathrm{mL}$ IFN-y to polarize them into classically activated macrophages (M1), or treated with $20 \mathrm{ng} / \mathrm{mL}$ IL-4 to polarize them into alternatively activated macrophages (M2).

Primary tumor-associated macrophage cells were isolated from the S-180 mouse sarcomas. Briefly, solid tumors were shredded and disaggregated by exposure to $0.3 \%$ collagenase IV (Invitrogen, CA, USA) for $40 \mathrm{~min}$ at $37^{\circ} \mathrm{C} ; 10^{6}$ cells $/ \mathrm{mL}$ were seeded in $60-\mathrm{mm}$ dishes (Corning, Tewksbury, MA). After $12 \mathrm{~h}$ of incubation, non-adherent cells were washed away, and adherent cells were maintained.

\section{MV isolation}

MVs were isolated from cell culture medium by differential centrifugation, according to previous publications (Valadi et al., 2007; Skog et al., 2008). After removing cells and other debris by centrifugation at $300 \mathrm{~g}$ and $3000 \mathrm{~g}$, the supernatant was centrifuged at $110,000 \mathrm{~g}$ for $70 \mathrm{~min}$ (all steps were performed at $4^{\circ} \mathrm{C}$ ). MVs were collected from the pellet and resuspended in PBS.

Cell transfection with scrambled negative control RNA, mimic-miR-150 RNA, and anti-miR-150 RNA

Synthetic RNA molecules, including mimic-miR-150, anti-miR-150, and scrambled negative control oligonucleotides (mimic-ncRNA or anti-ncRNA), were purchased from Ambion (Austin, TX). THP-1 cells and $293 \mathrm{~T}$ cells were seeded in $75 \mathrm{~cm}^{2}$ flasks (Corning) and were transfected the following day using Lipofectamine 2000 (Invitrogen), according to the manufacturer's instructions. To over-express miR-150 in 293T cells, 750 pmol of mimic-miR-150 RNA or scrambled negative control RNA was used. To knockdown miR-150, 750 pmol of antimiR-150 RNA or scrambled negative control anti-miRNA (anti-ncRNA) was used. Cells and media were harvested $48 \mathrm{~h}$ after transfection.

\section{RNA isolation and quantitative RT-PCR}

The total RNA of cells was extracted using TRIzol Reagent (Invitrogen), according to the manufacturer's recommended protocols. Quantitative RT-PCR of mature miRNA was performed on an ABI 7900 (Applied Biosystems; Foster City, CA) instrument using the Taqman miRNA probes (Applied Biosystems). Gene Quantitative RTPCR was performed on an $A B I 7900$ machine, and the primers used were purchased from Invitrogen. The primers used for macrophage marker genes were as follows: TNF-a: 5'-ACGGCATGGATCTCAAAGAC-3' (forward primer) and 5'-AGATAGCAAATCGGCTGACG-3' (reverse primer); Nos2: 5'-CCAAGCCCTCACCTACTTCC-3' (forward primer) and 5'-CTCTGAGGGCTGACACA-3' (reverse primer); Arg1: 5'-CTCCAAGCCAAAGTCCTTAGAG-3' (forward primer) and 5'-AGGAGCTGTCATTAGGGACATC-3' (reverse primer); and Msr2: 5'-AAAGAAAGCCCGAGTCCC-3' (forward primer) and 5'-TGCCCAAGGAGATAGCAAGA-3' (reverse primer).

\section{ELISA assay}

The VEGF levels in cell supernatants were measured using specific enzyme-linked immunosorbent assays. Briefly, $2 \mathrm{~mL}$ cell-free supernatant (each sample) from TAMs was harvested from $10^{5}$ cells after 8-hour incubations, and VEGF levels were determined using an ELISA kit (Invitrogen), following the manufacturer's instructions. Data were representative of 3 independent experiments.

\section{Western blotting}

The levels of VEGF protein in xenograft were quantified by Western blot analysis using antibodies against VEGF (147, Santa Cruz). Normalization was conducted by blotting the same samples with an antibody against GAPDH (6C5, Santa Cruz).

\section{Hematoxylin and eosin staining}

The tumors were fixed in $4 \%$ paraformaldehyde, embedded in paraffin, sectioned, and then stained with hematoxylin and eosin. To perform a quantitative analysis, the numbers of erythrocyte-filled blood vessels (capillary density) were counted in 5 independent fields from three sections.

\section{Immunohistochemistry}

The tumors were fixed in $4 \%$ paraformaldehyde, embedded in paraffin, sectioned, and then stained with DBE-conjugated anti-CD31 antibodies (Abcam, MA, USA). Quantitative analysis was conducted by quantifying the fluorescence intensity from five sections.

Statistical analysis

Statistical analysis was performed using a $T$-test. Data are presented 
as the means \pm SEM of at least three independent experiments. Differences were considered statistically significant at $P<0.05$.

\section{ACKNOWLEDGMENTS}

This work was supported by grants from the National Basic Research Program (973 Program) (No. 2014CB542300), the National Natural Science Foundation of China (Grant Nos. 31200969, 31200874, 81101330, 31271378, and 81250044), the National Science Fund for Distinguished Young Scholars (81025019), the Natural Science Foundation of Jiangsu Province (BK2011013 and BK2012014), and the Research Fund for the Doctoral Program of Higher Education of China (20120091120044).

\section{ABBREVIATIONS}

AVCS, angiogenic vascular cells; CAFs, cancer-associated fibroblastic cells; CSF-1, colony stimulating factor-1; HIF, hypoxia inducible factor; HRG, histidine-rich glycoprotein; IICs, infiltrating immune cells; MVs, microvesicles; TAMs, tumor-associated macrophages; TGF- $\beta$, transforming growth factor- $\beta$; TNF- $\alpha$, tumor necrosis factor; VEGF, vascular endothelial growth factor

\section{COMPLIANCE WITH ETHICS GUIDELINES}

Yuchen Liu, Luming Zhao, Dameng Li, Yuan Yin, Chen-Yu, Jing Li, Yujing Zhang declare that they have no conflict of interest. Additional informed consent was obtained from all patients for which identifying information is included in this article. All institutional and national guidelines for the care and use of laboratory animals were followed.

\section{REFERENCES}

Alvarez-Erviti, L., Seow, Y., Yin, H., Betts, C., Lakhal, S., and Wood, M.J. (2011). Delivery of siRNA to the mouse brain by systemic injection of targeted exosomes. Nat Biotechnol 29, 341-345.

Baek, J.H., Mahon, P.C., Oh, J., Kelly, B., Krishnamachary, B., Pearson, M., Chan, D.A., Giaccia, A.J., and Semenza, G.L. (2005). OS9 interacts with hypoxia-inducible factor 1alpha and prolyl hydroxylases to promote oxygen-dependent degradation of HIF-1alpha. Mol Cell 17, 503-512.

Bartel, D.P. (2004). MicroRNAs: genomics, biogenesis, mechanism, and function. Cell 116, 281-297.

Bingle, L., Brown, N.J., and Lewis, C.E. (2002). The role of tumourassociated macrophages in tumour progression: implications for new anticancer therapies. J Pathol 196, 254-265.

Bolat, F., Kayaselcuk, F., Nursal, T.Z., Yagmurdur, M.C., Bal, N., and Demirhan, B. (2006). Microvessel density, VEGF expression, and tumor-associated macrophages in breast tumors: correlations with prognostic parameters. J Exp Clin Cancer Res 25, 365-372.

Brahimi-Horn, C., and Pouyssegur, J. (2006). The role of the hypoxiainducible factor in tumor metabolism growth and invasion. Bulletin du cancer 93, E73-80.

Chen, X., Liang, H., Zhang, J., Zen, K., and Zhang, C.Y. (2012). Secreted microRNAs: a new form of i ntercellular communication. Trends Cell Biol 22, 125-132.

Coffelt, S.B., Hughes, R., and Lewis, C.E. (2009). Tumor-associated macrophages: effectors of angiogenesis and tumor progression. Biochim Biophys Acta 1796, 11-18.
Colla, S., Tagliaferri, S., Morandi, F., Lunghi, P., Donofrio, G., Martorana, D., Mancini, C., Lazzaretti, M., Mazzera, L., Ravanetti, L., et al. (2007). The new tumor-suppressor gene inhibitor of growth family member 4 (ING4) regulates the production of proangiogenic molecules by myeloma cells and suppresses hypoxia-inducible factor-1 alpha (HIF-1alpha) activity: involvement in myeloma-induced angiogenesis. Blood 110, 4464-4475.

Cristofanilli, M., Charnsangavej, C., and Hortobagyi, G.N. (2002). Angiogenesis modulation in cancer research: novel clinical approaches. Nat Rev Drug Discov 1, 415-426.

Esquela-Kerscher, A., and Slack, F.J. (2006). Oncomirs-microRNAs with a role in cancer. Nat Rev Cancer 6, 259-269.

Ferrara, N. (2010). Pathways mediating VEGF-independent tumor angiogenesis. Cytokine Growth Factor Rev 21, 21-26.

Ferrara, N., Gerber, H.P., and LeCouter, J. (2003). The biology of VEGF and its receptors. Nat Med 9, 669-676.

Folkman, J. (2007). Angiogenesis: an organizing principle for drug discovery? Nat Rev Drug Discov 6, 273-286.

Fukuda, K., Kobayashi, A., and Watabe, K. (2012). The role of tumorassociated macrophage in tumor progression. Front Biosci (Schol Ed) 4, 787-798.

Goga, A., and Benz, C. (2007). Anti-oncomir suppression of tumor phenotypes. Mol Interv 7, 199-202, 180.

Hanahan, D., and Coussens, L.M. (2012). Accessories to the crime: functions of cells recruited to the tumor microenvironment. Cancer Cell 21, 309-322.

Kim, K.J., Li, B., Winer, J., Armanini, M., Gillett, N., Phillips, H.S., and Ferrara, N. (1993). Inhibition of vascular endothelial growth factorinduced angiogenesis suppresses tumour growth in vivo. Nature 362, 841-844.

Kota, J., Chivukula, R.R., O'Donnell, K.A., Wentzel, E.A., Montgomery, C.L., Hwang, H.W., Chang, T.C., Vivekanandan, P., Torbenson, M., Clark, K.R., et al. (2009). Therapeutic microRNA delivery suppresses tumorigenesis in a murine liver cancer model. Cell 137, 1005-1017.

Lewis, C.E., Leek, R., Harris, A., and McGee, J.O. (1995). Cytokine regulation of angiogenesis in breast cancer: the role of tumorassociated macrophages. J Leukoc Biol 57, 747-751.

Lewis, C.E., and Pollard, J.W. (2006). Distinct role of macrophages in different tumor microenvironments. Cancer Res 66, 605-612.

Lin, E.Y., Nguyen, A.V., Russell, R.G., and Pollard, J.W. (2001). Colony-stimulating factor 1 promotes progression of mammary tumors to malignancy. J Exp Med 193, 727-740.

Lin, E.Y., and Pollard, J.W. (2007). Tumor-associated macrophages press the angiogenic switch in breast cancer. Cancer Res 67, 5064-5066.

Luo, Y., Zhou, H., Krueger, J., Kaplan, C., Lee, S.H., Dolman, C., Markowitz, D., Wu, W., Liu, C., Reisfeld, R.A., et al. (2006). Targeting tumor-associated macrophages as a novel strategy against breast cancer. J Clin Invest 116, 2132-2141.

McDonnell, C.O., Bouchier-Hayes, D.J., Toomey, D., Foley, D., Kay, E.W., Leen, E., and Walsh, T.N. (2003). Effect of neoadjuvant chemoradiotherapy on angiogenesis in oesophageal cancer. $\mathrm{Br} \mathrm{J}$ Surg 90, 1373-1378.

Millan Nunez-Cortes, J. (1991). Angiogenesis: a crucial element in tumor development. An Med Interna 8, 369-371.

Murdoch, C., Muthana, M., Coffelt, S.B., and Lewis, C.E. (2008). The 
role of myeloid cells in the promotion of tumour angiogenesis. Nat Rev Cancer 8, 618-631.

Ozer, A., Wu, L.C., and Bruick, R.K. (2005). The candidate tumor suppressor ING4 represses activation of the hypoxia inducible factor (HIF). Proc Natl Acad Sci U S A 102, 7481-7486.

Presta, L.G., Chen, H., O‘Connor, S.J., Chisholm, V., Meng, Y.G., Krummen, L., Winkler, M., and Ferrara, N. (1997). Humanization of an anti-vascular endothelial growth factor monoclonal antibody for the therapy of solid tumors and other disorders. Cancer Res 57, 4593-4599.

Qian, B.Z., and Pollard, J.W. (2010). Macrophage diversity enhances tumor progression and metastasis. Cell 141, 39-51.

Rolny, C., Mazzone, M., Tugues, S., Laoui, D., Johansson, I., Coulon, C., Squadrito, M.L., Segura, I., Li, X., Knevels, E., et al. (2011). HRG inhibits tumor growth and metastasis by inducing macrophage polarization and vessel normalization through downregulation of PIGF. Cancer Cell 19, 31-44.
Stockmann, C., Doedens, A., Weidemann, A., Zhang, N., Takeda, N., Greenberg, J.I., Cheresh, D.A., and Johnson, R.S. (2008). Deletion of vascular endothelial growth factor in myeloid cells accelerates tumorigenesis. Nature 456, 814-818.

Valadi, H., Ekstrom, K., Bossios, A., Sjostrand, M., Lee, J.J., and Lotvall, J.O. (2007). Exosome-mediated transfer of mRNAs and microRNAs is a novel mechanism of genetic exchange between cells. Nat Cell Biol 9, 654-659.

van den Boorn, J.G., Schlee, M., Coch, C., and Hartmann, G. (2011). SiRNA delivery with exosome nanoparticles. Nat Biotechnol 29, 325-326.

Warren, R.S., Yuan, H., Matli, M.R., Gillett, N.A., and Ferrara, N. (1995). Regulation by vascular endothelial growth factor of human colon cancer tumorigenesis in a mouse model of experimental liver metastasis. J Clin Invest 95, 1789-1797.

Zhang, Y., Liu, D., Chen, X., Li, J., Li, L., Bian, Z., Sun, F., Lu, J., Yin, Y., Cai, X., et al. (2010). Secreted monocytic miR-150 enhances targeted endothelial cell migration. Mol Cell 39, 133-144. 